\title{
Montréal hospitals preserve heritage
}

$\mathrm{T}$ his story begins in the dusty, slate-roofed attic of the Royal Victoria Hospital's Hersey Pavilion in Montréal. The former nurses' residence attic held artifacts squirrelled away over the decades by people who thought the artifacts might someday be important.

These items have a history of being transplanted. They came from the various hospitals of what is now the McGill University Health Centre (MUHC) in Montréal. Some reposed in the attic. Other items languished in corners of departments. Some filled closets in the homes of former doctors or nurses and their descendants, held onto for sentimental reasons, because of their beauty or uniqueness, or simply because they were overlooked.

Then another transplant: this one to harvest. The hospital community hauled in its items - the nursing school graduation portraits, fragile hand-blown $\mathrm{X}$-ray tubes, glass syringes stuck halfdrawn — to a dedicated central storage space awaiting sorting and redirection.

MUHC hospitals across the city, in full or in part, are moving to the new superhospital — the Glen site - in 2015. These institutions are part of the historic fabric of Montréal: the Royal Victoria opened in 1893, the Montreal Chest Institute began in 1903 and the Montreal Children's Hospital opened one year later. Some units of the Montreal General Hospital, founded in 1820, will also be moving to the Glen site.

The hospitals will each have their own spaces within the new building, but as they come together to form something new, they don't want to lose their past.

"A lot of people were concerned about what was going to happen to our history and to the objects that spoke of our history in clinical care and what we've contributed to the world in our field," says Karine Raynor, curator and associate director for the Art \& Heritage Centre of the MUHC, which is charged with preserving objects that show the hospitals' heritage.

Thousands of items have been collected, and thousands more are sure to trickle in. Meanwhile, the centre's team has to figure out how to display these items in the new hospital. In general terms, it's been decided that heritage items will be part of the overall healing environment at the new hospital, so that "art and culture and history could contribute to the patient ... [and] staff experience," Raynor says.

"It's not really a collection until it makes sense," she says. "You start to see the gaps ... and then you fill in those gaps. You can start to develop exhibition content and talk about particular themes and topics."

Right now, they know that they will

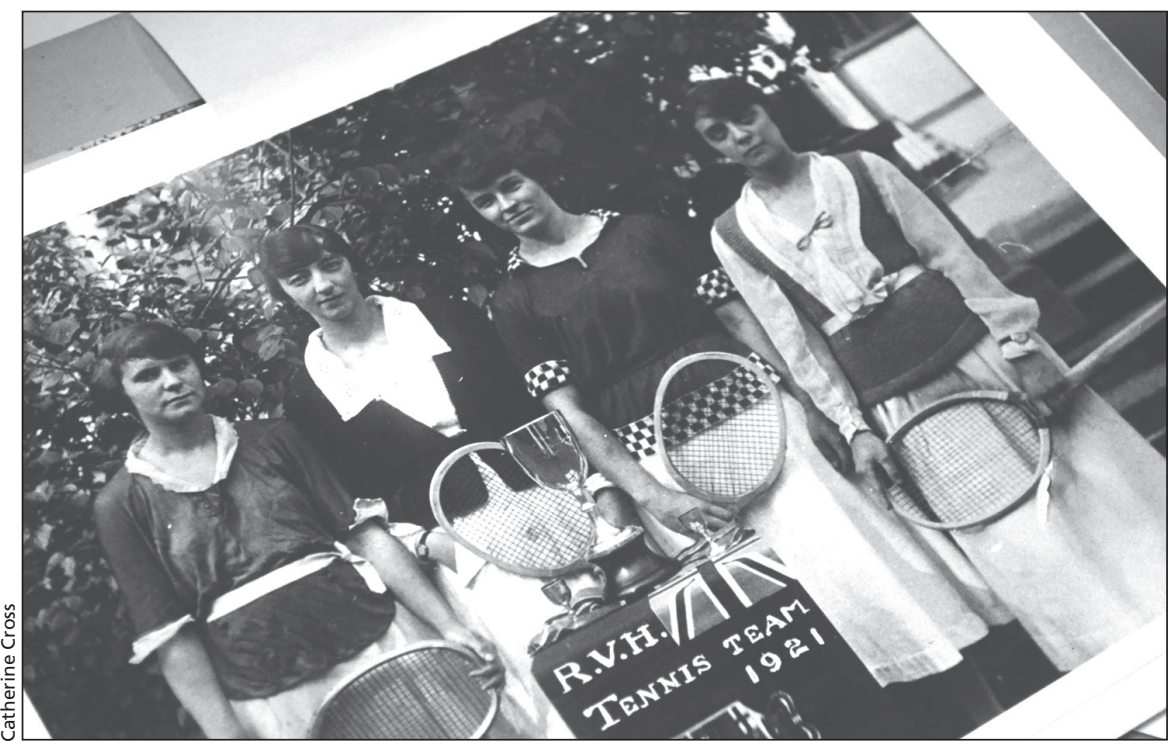

When nurses lived on-campus and had perhaps one night off every two weeks, the hospital social life was very important, and competitive sports teams were a frequent diversion. have over 100 glass cabinets of varying sizes throughout the halls of the new hospital, and they have no shortage of ideas when it comes to filling them.

"We can imagine having exhibitions of what a patient's tray looked like in 1894 , or what the place setting in the doctors' dining room might have been like in 1900," says Dr. Jonathan Meakins, a third-generation Royal Victoria Hospital physician and the Director of the Art \& Heritage Centre. The dishware and flatware would depict a more formal time; a time of high tea, silver cutlery, oyster spoons and butter knives.

Other cases might speak to the evolution of the treatment of diseases or technological developments in surgery. A display case could show, for example, technological advances in laparoscopic surgery, or how cataract surgery "has evolved from a two-week operation with a patient lying in bed with bricks beside their head [to ensure immobility] to an operation that's done in 20 minutes with the patient going home that afternoon," Meakins says.

The Art \& Heritage Centre team hopes these display cases, as well as art on the walls and sculptures, help visitors while away the hours. "It's kind of an oasis in the middle of a really busy and hectic space," says Raynor.

The process of consolidating the physical symbols of the hospitals' histories will also help ease the transition out of the old buildings and into the new, Art \& Heritage Centre, members hope.

Employees at all the hospitals have been invited to submit objects, which has created a momentum in the community, says Raynor. The exhibits themselves "will contribute to the sense of ownership of the new site."

Objects and documents that are not displayed will be stored in an archival space in the basement of one of the MUHC's office buildings. An archivist will help organize the records, and the team hopes to digitize at least some of the documents and photographs.

Beginning in the fall of 2014, each site will have its own exhibition, highlighting that institution's unique history. This is in preparation for the big transition - a transition not only in 


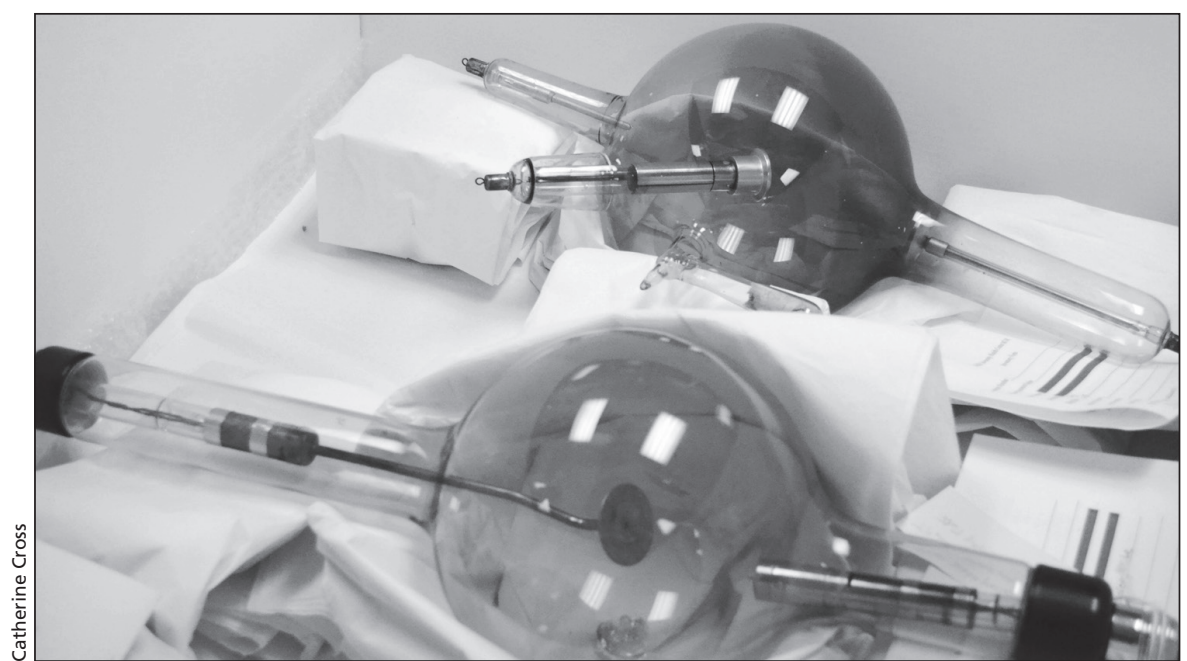

Skillfully hand-blown first- and second-generation X-ray tubes are carefully packed in a box. They may be used in exhibits to depict changes in X-ray technology over time.

terms of physical space, but also in culture and practice.

"Every hospital has their own history, but when you look at them from above you quickly realize that it's one history seen from different perspectives and all of those pieces fit into a puzzle. They complement each other and they speak to each other," says Raynor.

\section{Concerted effort}

The Art \& Heritage Centre was launched after several people, including Meakins and Raynor, wrote a document in 2010 alerting officials that in the absence of a concerted effort, a lot of things worth keeping would be thrown out.

The Art \& Heritage Centre was tasked with two gargantuan projects: to document what they had on hand, then figure out what to do with it. The collection is large, and has yet to be fully catalogued. So far, the Art \& Heritage Centre staff, assisted by volunteers, have inventoried 2000 objects, not including archival documents and images; they've yet to touch the Montreal Children's Hospital archive of roughly 15000 photos.

Many of the artifacts speak to the everyday life of hospitals: old hairbrushes, silver cutlery, a chamber pot, teacups, old packaged alcohol wipes and different types of syringes.

Medical equipment from yesteryear is always a crowd-pleaser, Meakins says. A pneumothorax apparatus was used to treat tuberculosis in the time before antibiotics. It collapsed a lung, in the hope that so doing would let the lung rest and heal.

The objects are only the beginning. The documents and photos will take a long time to sort through, and then there are paintings, sculptures and drawings.

Many of the photos in the collection from the Royal Victoria Hospital were taken by nurses and tell the stories of their training and lives. "For many years, nurses were required to live on the hospital site, with one night off every two weeks. If they were caught smoking, they were expelled," Raynor says.

"Some of our volunteers are retired nurses," says Alexandra Kirsh, the curatorial assistant. "As we go through the materials, they describe their experiences and how these have changed over time. They remember lining up every morning and having their head-to-toe check to make sure that everything was just so and starched precisely."

Medical residents were not held to the same standard as nurses in terms of stiff collars and cigarettes, but they too spent a lot of time at the hospital, which became a central part of their social life. Hospitals' sports teams competed against each other and many photos depict sports clubs and teams in competition or group shots.

Hospital culture may have changed since those times, but preserving the past is bringing people from across the MUHC network together to prepare for their future. - Catherine Cross, Ottawa, Ont. 